Research Paper

\title{
Multiple Antigen Stimulating Cellular Therapy (MASCT) For Hepatocellular Carcinoma After Curative Treatment: A Retrospective Study
}

\author{
Yajing $\mathrm{He}^{1}$, Yabing Guo ${ }^{1}$, Jinzhang Chen ${ }^{1}$, Xiaoyun $\mathrm{Hu}^{1}$, Xiaoshuang $\mathrm{Li}^{2}$, Yanjun Kong ${ }^{2}$, Xiaoyong Zhang1, \\ Xiangjun Zhou ${ }^{2}$, Li Liu ${ }^{1}$, Jinlin Hou ${ }^{\boxplus}$ \\ 1. State Key Laboratory of Organ Failure Research, Guangdong Provincial Key Laboratory of Viral Hepatitis Research, Department of Infectious Diseases, \\ Nanfang Hospital, Southern Medical University, Guangzhou, China. \\ 2. HRYZ Biotech Co., Shenzhen, China. \\ $\square$ Corresponding author: Li Liu, Professor, State Key Laboratory of Organ Failure Research, Guangdong Provincial Key Laboratory of Viral Hepatitis Research, \\ Department of Infectious Diseases, Nanfang Hospital, Southern Medical University, Guangzhou, China. e-mail: 18602062738@163.com, Tel: 18602062738. Jinlin \\ Hou, Professor, State Key Laboratory of Organ Failure Research, Guangdong Provincial Key Laboratory of Viral Hepatitis Research, Department of Infectious \\ Diseases, Nanfang Hospital, Southern Medical University, Guangzhou, China. e-mail: jlhousmu@163.com, Tel: 13802727354.
}

( $)$ Ivyspring International Publisher. This is an open access article distributed under the terms of the Creative Commons Attribution (CC BY-NC) license (https://creativecommons.org/licenses/by-nc/4.0/). See http://ivyspring.com/terms for full terms and conditions.

Received: 2017.11.07; Accepted: 2018.03.05; Published: 2018.04.06

\begin{abstract}
Background \& Aims: The prognosis of hepatocellular carcinoma $(\mathrm{HCC})$ remains poor and available treatment options are limited. This retrospective study evaluated the efficacy of Multiple Antigen Stimulating Cell Therapy (MASCT) as an adjuvant therapy for the treatment of HCC after curative treatment.

Methods: Patients who underwent HCC curative treatments were classified into two groups: the MASCT group, in which patients received MASCT treatment after curative treatment $(n=47)$, and the control group, in which patients did not receive any treatment after curative treatment $(n=99)$. Patients who received $\geq 5$ courses of MASCT treatment before recurrence or death $(n=26)$ were further stratified into a subgroup (multiple-course MASCT group) for analysis. The primary endpoint was overall survival (OS). The secondary endpoints were disease-free survival (DFS) and safety.

Results: Kaplan-Meier analysis showed no statistically significant difference in OS between the MASCT group and the control group $(P=0.132)$, nor in DFS $(P=0.310)$ (median: 36.17 vs. 24.27 months). However, when comparing the multiple-course MASCT treated group to the control group, Kaplan-Meier analysis showed a significant difference in OS $(P=0.011)$, but not in DFS $(P=0.104)$ (median: 47.10 vs. 24.27 months). The overall incidences of treatment-related adverse events in the MASCT group and control group were $14.89 \%$ (7/47) and 19.19\% (19/99), respectively. No MASCT treatment-related serious adverse events were reported.

Conclusions: Although the MASCT group was not associated with significantly longer OS or DFS, the multiple-course MASCT group showed significantly improved overall survival after curative treatment, and the treatment procedures were well-tolerated. Multiple-course MASCT may therefore provide another choice for patients with $\mathrm{HCC}$ after curative treatment.
\end{abstract}

Key words: hepatocellular carcinoma, dendritic cell vaccine, adoptive cell therapy, overall survival, disease-free survival.

\section{Introduction}

Hepatocellular carcinoma (HCC) is the fifth most common cancer in the world, accounting for $5.6 \%$ of all cancers. It is also the third most common cause of death due to cancer. Each year, nearly $82 \%$ of the approximately 550,000 liver cancer-related deaths occur in Asia, and $55 \%$ of all HCC cases worldwide occur in China ${ }^{1}$. Among the many risk factors identified, infection with the hepatitis B virus (HBV) is 
the cause of a large proportion of liver cancer cases (63.9\%; 65.9\% among men and 58.4\% among women $)^{2}$.

Although much progress has been made, in order to reduce death rates, HCC therapy still requires early diagnosis and treatment, and the postoperative outcome remains unsatisfactory. The 5 -year recurrence and survival rates after liver tumor resection are $68-73 \%$ and $34-81 \%$, respectively ${ }^{3-7}$. Indeed, HCC researchers are constantly aiming to find ways to prevent recurrence and improve prognosis in patients who have had curative treatment. In recent years, many studies have focused on postoperative adjuvant treatments, such as transcatheter arterial chemoembolization (TACE) $8-10$ and sorafenib ${ }^{11-12}$. However, the benefit of these therapies for postoperative adjuvant treatment remains unclear.

Cell-based cancer immunotherapies, including dendritic cell-based therapeutic cancer vaccines13 and adoptive cell therapies (ACT) ${ }^{14}$, have attracted great attention in the clinical treatment of tumors in recent years. As the most potent antigen presenting cell (APC), DCs from the patient's peripheral blood mononuclear cells (PBMCs) can be loaded with different forms of tumor antigens, activated in vitro, and then injected into patients, therefore inducing the expansion of circulating CD4+ $\mathrm{T}$ cells and CD8+ T cells that are specific for tumor antigens ${ }^{15-17}$. ACTbased immunotherapies, such as chimeric antigen receptors (CARs)-engineered $\mathrm{T}$ cell therapy, have shown exciting clinical benefits in diverse types of cancers $^{14}$, 18-19. Multiple antigen stimulating cell therapy (MASCT), as the first application to combine $\mathrm{DC}$ vaccine and adoptive $\mathrm{T}$ cell transfer in one treatment, has been used to treat patients with different phases of HCC in our clinic. We have previously demonstrated that $\mathrm{T}$ cells against multiple antigens can be induced and proliferated in vivo by MASCT for patients after liver tumor resection ${ }^{20}$. In the present study, we retrospectively investigated the efficacy and safety of MASCT for HCC patients after curative treatment.

\section{Materials and Methods}

\section{Patients}

All the patients diagnosed as HCC in the Hepatology Unit of Nanfang Hospital from July 2011 to July 2015 were screened in this study consecutively. Patients received only symptomatic treatment or traditional Chinese medicine were not recorded. As recorded, 974 patients had received liver tumor treatment during this period, including operation, percutaneous ablation, TACE, sorafenib, radiotherapy, and chemotherapy. Among them, 272 patients had received curative treatment. HCC diagnosis was established according to the European Association for the Study of the Liver (EASL) ${ }^{21}$ HCC management guidelines. The collection of clinical data was approved by the Ethics Committee of Nanfang Hospital.

The inclusion criteria of the study were: (1) treated with curative therapy between July 2011 and July 2015, with regular follow-ups; (2) treated with MASCT only, or no treatment, during the period from curative treatment to recurrence or death; (3) Barcelona Clinic Liver Cancer (BCLC) stage 0-B; (4) Child-Pugh liver function stage A-B; (5) Karnofsky (KPS) score 70-100; (6) life expectancy of $>3$ months.

The exclusion criteria were: (1) diagnosis of recurrence within 2 months of curative treatment, confirmed by computed tomography (CT) or magnetic resonance imaging (MRI); (2) treatment with other adjuvant therapies (e.g. TACE) besides MASCT during the period from curative treatment to recurrence; (3) treatment with MASCT outside of the period from curative treatment to recurrence or death; (4) presence of other tumors at the time of HCC diagnosis or during the follow-up period; (5) BCLC stage C-D; (6) curative treatment with liver transplantation, or accompanied by immune deficiency or autoimmune diseases; (7) advanced liver disease (as shown by Child-Pugh stage C liver function), gastrointestinal bleeding, encephalopathy, ascites, or other serious complications that were not compatible with HCC treatment.

A total of 974 patients were screened and 146 patients (who met all of inclusion criteria with none of the exclusion criteria) were included in the study. Of these, 47 patients received MASCT and 99 did not. Screening details and patient dispositions are shown in Figure 1.

Baseline demographics, such as gender and age, and clinical information were recorded, including: Child-Pugh stage, BCLC stage, tumor burden (size and number), HBV infection, HBV DNA, cirrhosis, AFP, blood test results (WBC, LYM, PLT, ALP, GGT, TBIL, ALT, AST, ALB, PT), time(s) of MASCT infusion, OS, and DFS.

\section{Study Protocol}

Based on their adjuvant treatment after curative treatment and before recurrence, HCC patients were classified into two groups: the MASCT group, in which patients received MASCT $(n=47)$, and the control group, in which patients did not receive any adjuvant treatment $(n=99)$. Patients who received $\geq 5$ courses of MASCT before recurrence $(n=26)$ were further stratified into the multiple-course MASCT subgroup. The patients with OS $<5$ months in the multiple-course MASCT subgroup were excluded. 


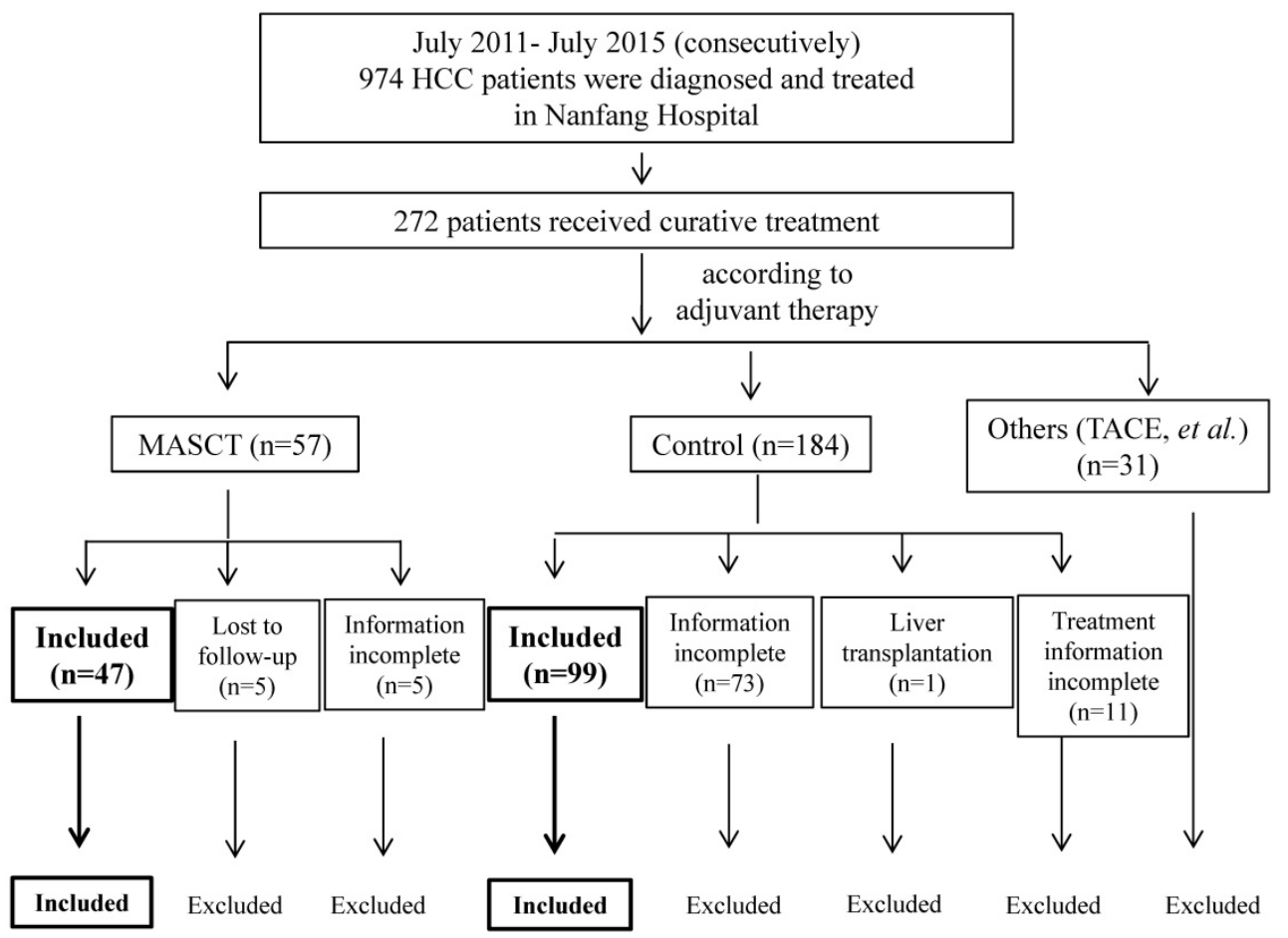

Figure 1. Patient disposition

Curative treatment consisted of either surgical resection or percutaneous ablation therapies, including radiofrequency (RF), cryoablation, and percutaneous alcohol injection (PEI) ${ }^{22,23}$.

Table 1. Composition and characteristics of the HCC antigen

\begin{tabular}{lll}
\hline No. & Antigen & Overexpressed in HCC \\
\hline 1 & hTERT & + \\
2 & P53 & + (loss of function) \\
3 & Suvivin & + \\
4 & NY-ESO-1 & + \\
5 & CEA & + \\
6 & CCND1 & + \\
7 & c-MET & + \\
8 & RGS5 & + \\
9 & MMP7 & + \\
10 & VEGFR & + \\
11 & AFP & + \\
12 & GPC3 & $+\left(\right.$ when $\left.\mathrm{HBV}^{+}\right)$ \\
13 & HBV core antigen & $+\left(\right.$ when $\left.\mathrm{HBV}^{+}\right)$ \\
14 & HBV DNA polymerase &
\end{tabular}

\section{Treatment Procedures}

\section{Cell Preparation for MASCT}

The cell preparation procedure for MASCT was identical to our published study ${ }^{20}$. The patients underwent leukapheresis and approximately $2 \times 10^{9}$ mononuclear cells were collected. The cells were then subjected to density gradient centrifugation using lymphoprep (Nycomed Pharma, Oslo, Norway) to remove the red blood cells, which were contaminated during the leukapheresis process. PBMCs were then seeded into three $225 \mathrm{~cm}^{2}$ culture flasks and were kept at $37{ }^{\circ} \mathrm{C}$ in a saturated $5 \%$ $\mathrm{CO} 2$ incubator for 1.5 hours. Afterwards, the adherent monocytes were cultured in AIM-V medium with 500 $\mathrm{U} / \mathrm{mL}$ IL-4 and $1000 \mathrm{U} / \mathrm{mL}$ GM-CSF, to differentiate into immature DCs. The immature DCs were pulsed by a peptide pool of multiple tumor antigens (1 $\mu \mathrm{g} / \mathrm{ml} /$ peptide), followed by culture with a maturing cocktail (IL-6, $1000 \mathrm{U} / \mathrm{ml}$; TNF-a, $1000 \mathrm{U} / \mathrm{ml}$; IL-1ß, $10000 \mathrm{U} / \mathrm{ml}$; PEG2, $1 \mu \mathrm{g} / \mathrm{ml}$; Poly I:C, $10 \mu \mathrm{g} / \mathrm{ml}$ ), to differentiate into antigen-presenting mature DCs. The peptide pool of multiple tumor antigens used in MASCT involved 14 antigens, including 12 tumor-associated antigens (TAAs) overexpressed in cancerous hepatocytes (hTERT, p53, survivin, NY-ESO-1, CEA, CCND1, c-MET, RGS5, MMP7, VEGFR, AFP, GPC3), and $2 \mathrm{HBV}$-associated antigens (HBV core antigen, HBV DNA polymerase) (Table 1). A portion of the mature DCs were injected to patients subcutaneously, and the remaining DCs were further divided into two equal parts. One part was frozen for the second subcutaneous injection, and the other part was co-cultured with non-adherent cells for the preparation of active $\mathrm{T}$ cells. To prepare the activated $\mathrm{T}$ cells for infusion, the frozen non-adherent PBMCs were co-cultured with antigen-loaded mature DCs. The anti-CD3 antibody (50 ng/ml; eBioscience, Inc., San Diego, CA) was added 3 days after co-culturing, then cells were cultured for about 3 weeks in the presence of IL-2 $(1000 \mathrm{U} / \mathrm{ml}$; R\&D Systems Inc, Minneapolis, MN). Each patient received approximately $6 \times 10^{9}$ active $T$ cells per infusion. 
Every patient underwent leukapheresis once, and two blood draws were taken during a treatment course. The PBMCs collected from each leukapheresis and blood sample were used to prepare cells for 2 DC subcutaneous injections and 3 active $T$ cell infusions. Two DC injections were administered to patients with a two-week interval in the first month of the course, and the active $\mathrm{T}$ cell infusions were administered to patients every four weeks. The course was calculated on the infusion of MASCT.

\section{Follow-up and Outcome Measures}

Follow-up began at the time of curative treatment and ended at the time of death or at the last follow-up. Data regarding the disease and survival statuses of the patients were collected every 3 months in the first 2 years, and every 6 months thereafter.

The primary endpoint was overall survival (OS), which was measured from the date of curative treatment, to the time of death or the last follow-up. The secondary endpoints were disease-free survival (DFS) and safety. DFS was measured from the date of curative treatment, to either the time of first recurrence or to the date of the last follow-up (for patients with no recurrence). Tumor assessments were performed using enhanced CT or MRI every 2-3 months. All scans were evaluated by two independent radiologists on site, who had more than 5 years of experience. Safety was assessed using version 4.03 of the National Cancer Institute's Common Terminology Criteria for adverse events (CTCAE 3.0) ${ }^{24}$.

\section{Statistical Analysis}

Continuous data was expressed as mean values $\pm \mathrm{SD}$, or as median values (P25-P75) as appropriate. Discrete data was expressed as $\mathrm{n}$ (percentage). Quantitative data in normal distributions were compared by t-test, while quantitative data in non-normal distributions were compared using the Wilcoxon rank-sum test. Qualitative data were compared using Pearson's chi-square test or Fisher's exact test as appropriate. The Kaplan-Meier method was used to estimate the survival curve for each prognostic group. The log-rank test was used to test whether significant survival differences were present between different prognostic groups. A cox proportional hazard analysis was performed to assess the effect of baseline characteristics on each outcome of interest. Hazard ratios (HRs), as well as their confidence intervals, were estimated using the Cox proportional hazards model. Adverse events (AEs) were compared using either the chi-square test or the Fisher exact test. Survival time was presented as the median with a 95\% confidence interval (CI). All statistical tests were 2 -sided and a $\mathrm{P}$ value $<0.05$ was considered statistically significant. All statistical analyses were performed using SPSS version 20.0 (SPSS Inc., Chicago, IL, USA).

\section{Results}

\section{Clinical Characteristics of Patients}

Of the 146 patients included in this study, 122 $(83.6 \%)$ were male and $24(16.4 \%)$ were female. The mean patient age was 51.94 years (range: $16-82$ years). Most of the patients were affected by HBV infection $(90.4 \%)$, cirrhosis $(89.7 \%)$, and good liver function (Child-Pugh Stage A) (84.2\%). The patient distribution of BCLC stage was as follows: stage 0-A, $55.5 \%$; stage B, $44.5 \%$. The median follow-up times for the MASCT group and the control group were 34.97 months and 30.50 months, respectively. Most of the patients (46/47 in the MASCT group and 98/99 in the control group) died due to HCC recurrence and progression. In the MASCT group, the median number of MASCT infusions was 6 (range: 1 - 15). Eight patients were discontinued from MASCT treatment after tumor recurrence.

The baseline characteristic of age was significantly different between the two groups. Compared with the control group, patients in the MASCT group were young (Table 2). Similarly, compared with the control group, patients in the multiple-course MASCT subgroup were young (Table $3)$.

\section{Efficacy in the MASCT Group Versus the Control Group}

\section{Overall Survival}

At the time of data cut-off, 8 patients $(17.0 \%)$ in the MASCT group and 28 patients $(28.3 \%)$ in the control group had died. There were no treatment-related deaths. The median survival times in both the MASCT group and the control group were not reached. The cumulative survival rates at 1,3 , and 5 years were $95.7 \%, 85.1 \%$, and $75.6 \%$ in the MASCT group, and $92.9 \%, 73.2 \%$, and $62.8 \%$ in the control group, respectively (Table 4). The OS rates of the two groups were not significantly different $(P=0.132$ by log-rank test) (Figure 2A). The HR for death in the MASCT group vs. the control group was 0.551 (95\% CI: $0.251-1.209)$, representing a $44.9 \%$ relative risk reduction in the MASCT group compared with the control group.

Upon multivariate analysis with a stepwise selection method, tumor size and alkaline phosphatase (ALP) were independent predictors of OS for HCC treatment (Table S1.). 

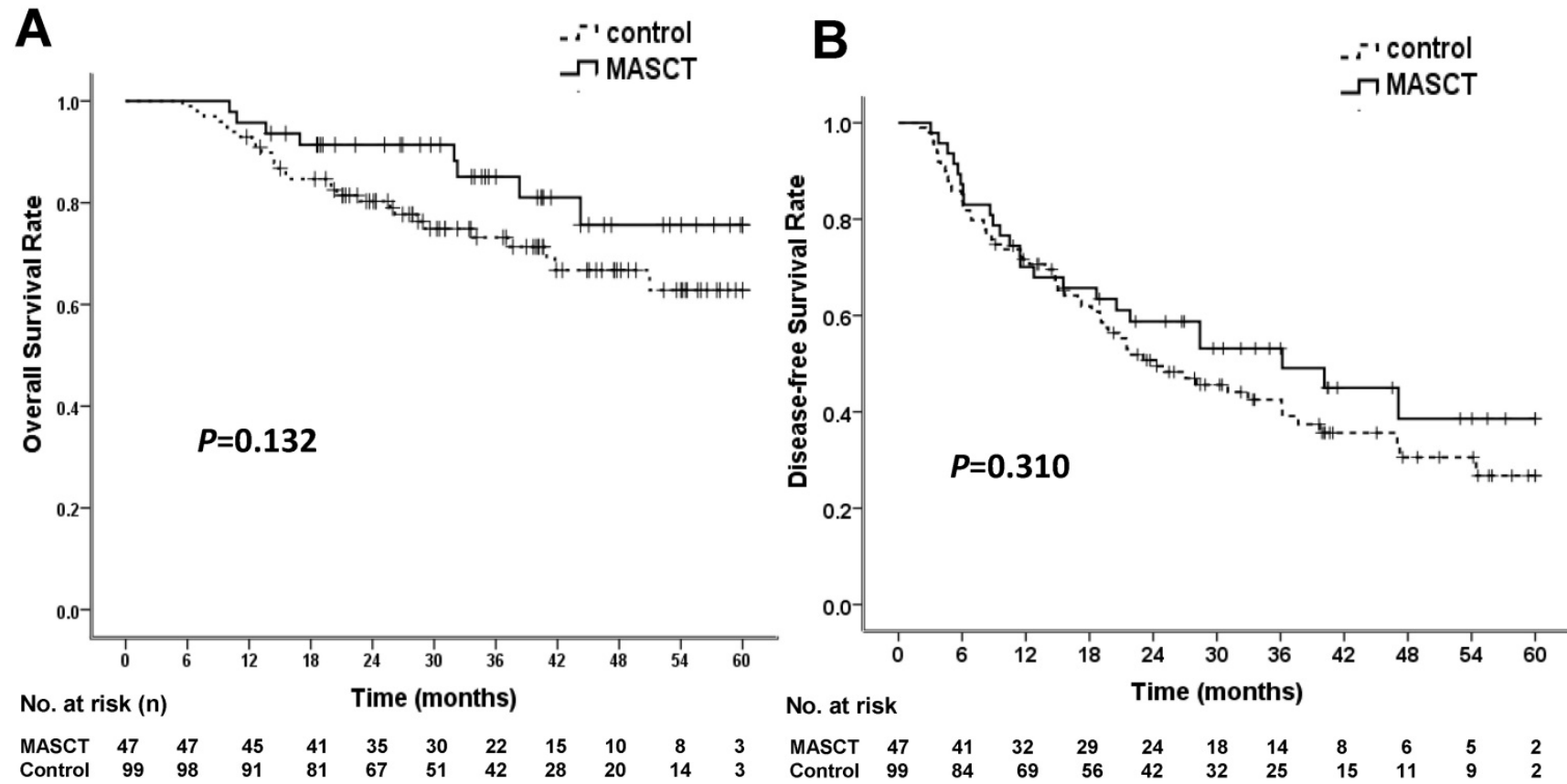

$\begin{array}{lccccccccccccccccccccccc}\text { MASCT } & 47 & 47 & 45 & 41 & 35 & 30 & 22 & 15 & 10 & 8 & 3 & \text { MASCT } & 47 & 41 & 32 & 29 & 24 & 18 & 14 & 8 & 6 & 5 & 2 \\ \text { Control } & 99 & 98 & 91 & 81 & 67 & 51 & 42 & 28 & 20 & 14 & 3 & \text { Control } & 99 & 84 & 69 & 56 & 42 & 32 & 25 & 15 & 11 & 9 & 2\end{array}$

Figure 2. Overall survival (OS) and disease-free survival (DFS) rates in MASCT and control groups. (A) OS curve showed no significant difference. (B) DFS curve showed no significant difference

Table 2. Baseline characteristics of patients in the MASCT group and control group.

\begin{tabular}{|c|c|c|c|c|}
\hline Variables (N; \%) & $\begin{array}{l}\text { All patients } \\
(\mathrm{n}=146)\end{array}$ & $\begin{array}{l}\text { MASCT group } \\
(\mathrm{n}=47)\end{array}$ & $\begin{array}{l}\text { Control group } \\
(\mathrm{n}=99)\end{array}$ & $\begin{array}{l}P \\
\text { value }\end{array}$ \\
\hline Gender & & & & 0.896 \\
\hline Male & $122(83.6)$ & $39(83.0)$ & $83(83.8)$ & \\
\hline Female & $24(16.4)$ & $8(17.0)$ & $16(16.2)$ & \\
\hline Age, Mean \pm SD & $51.94 \pm 11.90$ & $48.60 \pm 12.60$ & $53.53 \pm 11.26$ & 0.019 \\
\hline Etiology & & & & 0.550 \\
\hline HBV infection only & $132(90.4)$ & $41(87.2)$ & $91(91.9)$ & \\
\hline $\begin{array}{l}\mathrm{HCV}, \mathrm{HBV} \text { and } \mathrm{HCV} \\
\text { coinfection, others }\end{array}$ & $14(9.6)$ & $6(12.8)$ & $8(8.1)$ & \\
\hline Cirrhosis & & & & 0.848 \\
\hline Yes & $131(89.7)$ & $43(91.5)$ & $88(88.9)$ & \\
\hline No & $15(10.3)$ & $4(8.5)$ & $11(11.1)$ & \\
\hline Child-Pugh Stage & & & & 0.844 \\
\hline A & $123(84.2)$ & $40(85.1)$ & $83(83.8)$ & \\
\hline B & $23(15.8)$ & 7 (14.9) & $16(16.2)$ & \\
\hline BCLC Stage & & & & 0.351 \\
\hline $0-\mathrm{A}$ & $133(91.1)$ & $41(87.2)$ & $92(92.9)$ & \\
\hline B & $13(8.9)$ & $6(12.8)$ & $7(7.1)$ & \\
\hline Tumor number & & & & 0.421 \\
\hline 1 & $126(86.3)$ & $39(83.0)$ & 87 (87.9) & \\
\hline$\geq 2$ & $20(13.7)$ & $8(6.4)$ & $12(12.1)$ & \\
\hline Tumor size & & & & 0.334 \\
\hline$\leq 3 \mathrm{~cm}$ & $86(58.9)$ & $25(53.2)$ & $61(61.6)$ & \\
\hline$>3 \mathrm{~cm}$ & $60(41.1)$ & $22(46.8)$ & $38(38.4)$ & \\
\hline $\mathrm{AFP}^{*}$, Median $(25 \%, 75 \%)$ & $\begin{array}{l}45.08(6.24, \\
414.83)\end{array}$ & $\begin{array}{l}18.69 \\
(4.18,465.16)\end{array}$ & $\begin{array}{l}73.85(9.23 \\
414.67)\end{array}$ & 0.169 \\
\hline Antiviral therapy & & & & 0.330 \\
\hline Yes & $131(89.7)$ & $40(85.1)$ & $91(91.9)$ & \\
\hline No & $15(10.3)$ & 7 (14.9) & $8(8.1)$ & \\
\hline $\begin{array}{l}\text { Curative treatment } \\
\text { modality }\end{array}$ & & & & 0.850 \\
\hline Liver resection & $70(47.9)$ & $22(46.8)$ & $48(48.5)$ & \\
\hline Percutaneous ablation & $76(52.1)$ & $25(53.2)$ & $51(51.5)$ & \\
\hline
\end{tabular}

Table 3. Baseline characteristics of patients in the multiple-course MASCT subgroup and control group.

\begin{tabular}{|c|c|c|c|c|}
\hline Variables $(\mathrm{N} ; \%)$ & $\begin{array}{l}\text { All } \\
\text { patients } \\
(n=125)\end{array}$ & $\begin{array}{l}\text { Multiple courses of } \\
\text { MASCT group } \\
(\mathrm{n}=26)\end{array}$ & $\begin{array}{l}\text { Control } \\
\text { group } \\
(n=99)\end{array}$ & $\begin{array}{l}P \\
\text { value }\end{array}$ \\
\hline Gender & & & & 0.938 \\
\hline Male & $104(83.2)$ & $21(80.8)$ & $83(83.8)$ & \\
\hline Female & $21(16.8)$ & $5(19.2)$ & $16(16.2)$ & \\
\hline Age, Mean \pm SD & $\begin{array}{l}51.97 \pm \\
11.42\end{array}$ & $46.04 \pm 10.16$ & $\begin{array}{l}53.53 \pm \\
11.26\end{array}$ & 0.003 \\
\hline Etiology & & & & 1.000 \\
\hline HBV infection only & $115(92.0)$ & $24(92.3)$ & $91(91.9)$ & \\
\hline $\begin{array}{l}\mathrm{HCV}, \mathrm{HBV} \text { and } \mathrm{HCV} \\
\text { coinfection, others }\end{array}$ & $10(8.0)$ & $2(7.7)$ & $8(8.1)$ & \\
\hline Cirrhosis & & & & 0.456 \\
\hline Yes & $113(90.4)$ & $25(96.2)$ & $88(88.9)$ & \\
\hline No & $12(9.6)$ & $1(3.8)$ & $11(11.1)$ & \\
\hline Child-Pugh Stage & & & & 0.191 \\
\hline A & $108(86.4)$ & $25(96.2)$ & $83(83.8)$ & \\
\hline B & 17 (13.6) & $1(3.8)$ & $16(16.2)$ & \\
\hline BCLC Stage & & & & 0.238 \\
\hline $0-\mathrm{A}$ & $114(91.2)$ & $22(84.6)$ & $92(92.9)$ & \\
\hline B & $11(8.8)$ & $4(15.4)$ & $7(7.1)$ & \\
\hline Tumor number & & & & 0.346 \\
\hline 1 & $108(86.4)$ & $21(80.8)$ & 87 (87.9) & \\
\hline$\geq 2$ & 17 (13.6) & $5(19.2)$ & $12(12.1)$ & \\
\hline Tumor size & & & & 0.472 \\
\hline$\leq 3 \mathrm{~cm}$ & $75(60.0)$ & $14(53.8)$ & $61(61.6)$ & \\
\hline$>3 \mathrm{~cm}$ & $50(40.0)$ & $12(46.2)$ & $38(38.4)$ & \\
\hline $\begin{array}{l}\mathrm{AFP} * \text {, Median }(25 \%, \\
75 \%)\end{array}$ & $\begin{array}{l}73.81(8.36, \\
433.60)\end{array}$ & $64.85(4.26,1365.63)$ & $\begin{array}{l}73.85(9.23 \\
414.67)\end{array}$ & 0.804 \\
\hline Antiviral therapy & & & & 0.869 \\
\hline Yes & $114(91.2)$ & $23(88.5)$ & $91(91.9)$ & \\
\hline No & $11(8.8)$ & $3(11.5)$ & $8(8.1)$ & \\
\hline $\begin{array}{l}\text { Curative treatment } \\
\text { modality }\end{array}$ & & & & 0.403 \\
\hline Liver resection & $63(50.4)$ & $15(57.7)$ & $48(48.5)$ & \\
\hline Percutaneous ablation & $62(49.6)$ & $11(42.3)$ & $51(51.5)$ & \\
\hline
\end{tabular}


Table 4. Survival analysis of patients in the MASCT group and control group.

\begin{tabular}{llll}
\hline & $\begin{array}{l}\text { MASCT group }(\mathbf{n}= \\
\text { 47) }\end{array}$ & Control group $(\mathbf{n}=\mathbf{9 9})$ & $\begin{array}{l}\boldsymbol{P} \\
\text { value }\end{array}$ \\
\hline OS (median, 95\% CI) (mo) & Not reached & Not reached & 0.132 \\
OS rate (\%) & - & - & - \\
1 y & 95.7 & 92.9 & \\
3 y & 85.1 & 73.2 & \\
5 y & 75.6 & 62.8 & 0.310 \\
DFS (median, 95\% CI) & 36.167 (15.235-57.098) & 24.267 (14.937-33.597) & \\
(mo) & & & - \\
DFS rate (\%) & - & - & \\
1 y & 70.1 & 71.7 & \\
3 y & 53.1 & 42.5 & \\
5 y & 38.5 & 26.7 & \\
\hline Abbreviations: CI: confidence interval; MASCT: multiple antigen stimulating \\
cellular therapy; mo: month; OS: overall survival; DFS: disease-free survival; y: \\
year.
\end{tabular}

Table 5. Survival analysis of patients between multiple courses of MASCT group and control group.

\begin{tabular}{llll}
\hline & $\begin{array}{l}\text { Multiple courses of MASCT } \\
\text { group (n= 26) }\end{array}$ & $\begin{array}{l}\text { Control group (n= } \\
\mathbf{9 9})\end{array}$ & $\begin{array}{l}\boldsymbol{P} \\
\text { value }\end{array}$ \\
\hline OS (median, 95\% & Not reached & Not reached & 0.011 \\
CI) (mo) & & & - \\
OS rate (\%) & - & - & \\
1 y & 100 & 92.9 & \\
3 y & 100 & 73.2 & 0.104 \\
5 y & 92.9 & 62.8 & - \\
DFS (median, 95\% & $47.100(25.055-69.145)$ & 24.267 & \\
CI) (mo) & & $(14.937-33.597)$ & \\
DFS rate (\%) & - & - & \\
1 y & 76.9 & 71.7 & \\
3 y & 65.4 & 42.5 & \\
5 y & 43.6 & 26.7 & \\
\hline Abbreviations: CI: confidence interval; MASCT: multiple antigen stimulating \\
cellular therapy; OS: overall survival; DFS: disease-free survival; y: year.
\end{tabular}

Disease-free Survival

At the time of data cut-off, 24 patients $(51.1 \%)$ in the MASCT group and 60 patients $(60.6 \%)$ in the control group experienced tumor recurrence. The median DFS times in the MASCT and control groups were $36.17 \pm 10.68$ months (95\% CI: 15.24 - 57.10 months) and $24.27 \pm 4.76$ months (95\% CI: $14.94-$ 33.60 months), respectively. The cumulative recurrence rates at 1,3 , and 5 years were $29.9 \%, 46.9 \%$, and $61.5 \%$ in the MASCT group, and $28.3 \%, 57.5 \%$, and $73.3 \%$ in the control group, respectively (Table 4). The two groups were not significantly different in terms of DFS ( $\mathrm{P}=0.310$ by log-rank test) (Figure $2 \mathrm{~B})$. The HR for recurrence of the MASCT group vs. the control group was 0.783 (95\% CI: 0.488 - 1.257), representing a $21.7 \%$ relative risk reduction in the MASCT group compared with the control group.

\section{Efficacy in the Multiple-Course MASCT Subgroup Versus the Control Group}

\section{Overall Survival}

In the multiple-course MASCT subgroup, all 26 patients received $\geq 5$ courses of MASCT before death. At the time of data cut-off, 1 patient $(3.8 \%)$ in the multiple-course MASCT subgroup, and 28 patients $(28.3 \%)$ in the control group had died. The median survival times in both the multiple-course MASCT subgroup and the control group were not reached. The cumulative survival rates at 1,3 , and 5 years were $100 \%, 100 \%$, and $92.9 \%$ in the multiple-course MASCT group, and $92.9 \%, 73.2 \%$, and $62.8 \%$ in the control group, respectively (Table 5). The OS rate in the multiple-course MASCT group was significantly higher than that in the control group $(P=0.011$ by log-rank test) (Figure 3A). The HR for death in the multiple-course MASCT group vs. the control group was 0.116 (95\% CI: 0.016 - 0.852), representing an $88.4 \%$ relative risk reduction in the multiple-course MASCT group compared with the control group.

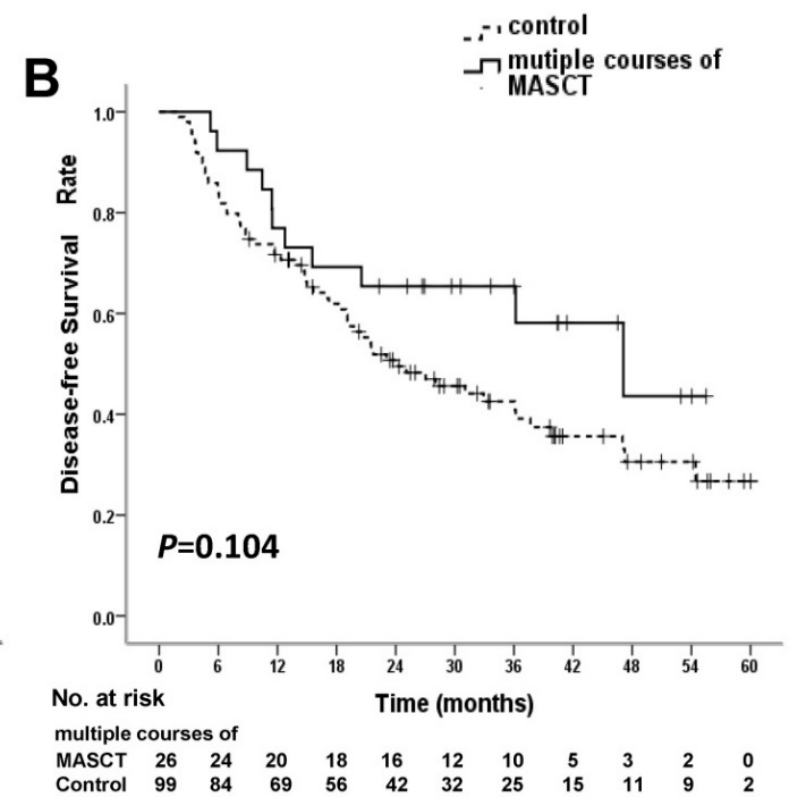

Figure 3. Overall survival (OS) and disease-free survival (DFS) rates in multiple-course MASCT subgroup and control group. (A) OS curve for the multiple-course MASCT subgroup was statistically significantly superior to that for the control group. (B) DFS curves showed no significant difference 
Multivariate analysis using a stepwise selection method indicated that multiple-course MASCT treatment was a significant prognostic factor for OS (HR: 0.055, 95\% CI: 0.007 - 0.445). Besides MASCT, tumor size, ALP and albumin (ALB) were also independent predictors of OS for HCC treatment (Table S2.).

\section{Disease-free Survival}

At the time of data cut-off, 11 patients (42.3\%) in the multiple-course MASCT group and 60 patients $(60.6 \%)$ in the control group experienced tumor recurrence. The median DFS times in the multiple-course MASCT group and the control group were $47.10 \pm 11.25$ months (95\% CI: 25.06 - 69.15 months) and $24.27 \pm 4.76$ months (95\% CI: $14.94-$ 33.60 months), respectively. The cumulative recurrence rates at 1,3 , and 5 years were $23.1 \%, 34.6 \%$, and $56.4 \%$ in the multiple-course MASCT group, and $28.3 \%, 57.5 \%$, and $73.3 \%$ in the control group, respectively (Table 5). The two groups were not significantly different in terms of DFS rate $(P=0.104$ by log-rank test) (Figure 3B). The HR for recurrence in the multiple-course MASCT group vs. the control group was 0.590 (95\% CI: 0.310-1.123), representing a $41.0 \%$ relative risk reduction in the multiple-course MASCT group compared with the control group.

\section{Safety}

Overall, AEs were reported for 26 patients $(17.81 \%)$. No serious adverse event (SAE) was reported during the procedures. The overall incidences of treatment-related AEs were 14.89\% $(7 / 47)$ in the MASCT group, and $19.19 \%$ (19/99) in the control group. There was no significant difference between the two groups $(P=0.526)$. Overall, the most common adverse events were fever (12 patients, $8.22 \%$ ) and flu-like symptoms (8 patients, $5.48 \%$ ) (Table 6).

Table 6. Adverse events in patients who received MASCT and the control group.

\begin{tabular}{|c|c|c|c|c|c|c|}
\hline \multirow{2}{*}{$\begin{array}{l}\text { Adverse } \\
\text { event }\end{array}$} & \multicolumn{2}{|c|}{ All patients $(n=146)$} & \multicolumn{2}{|c|}{ MASCT $(n=47)$} & \multicolumn{2}{|c|}{ Control $(n=99)$} \\
\hline & Grade 1 & Grade 2 & Grade 1 & Grade 2 & Grade 1 & Grade 2 \\
\hline $\begin{array}{l}\text { Overall } \\
\text { incidence }\end{array}$ & 18 & 8 & 5 & 2 & 13 & 6 \\
\hline Fever & 9 & 3 & 2 & 1 & 7 & 2 \\
\hline $\begin{array}{l}\text { Flu-like } \\
\text { symptoms }\end{array}$ & 5 & 3 & 1 & 0 & 4 & 3 \\
\hline $\begin{array}{l}\text { Liver } \\
\text { dysfunction }\end{array}$ & 1 & 1 & 1 & 0 & 0 & 1 \\
\hline $\begin{array}{l}\text { Allergic } \\
\text { reaction }\end{array}$ & 0 & 1 & 0 & 1 & 0 & 0 \\
\hline $\begin{array}{l}\text { Abdominal } \\
\text { pain }\end{array}$ & 1 & 0 & 1 & 0 & 0 & 0 \\
\hline Ascites & 1 & 0 & 0 & 0 & 1 & 0 \\
\hline Osteodynia & 1 & 0 & 0 & 0 & 1 & 0 \\
\hline
\end{tabular}

\section{Discussion}

In this study, the median DFS was 11.90 months longer for patients in the MASCT group compared with patients in the control group. Nevertheless, when comparing these two treatment groups, there were no statistically significant differences in OS $(P=$ $0.132)$ and DFS $(P=0.310)$. For patients in the multiple-course MASCT treated group (those who received $\geq 5$ courses MASCT), the median DFS benefit was 22.83 months longer than that of the control group. The difference in OS between these two groups was significant $(P=0.011)$, although the difference in DFS was not $(P=0.104)$. These results suggest that the survival of patients receiving MASCT, especially in patients who received $\geq 5$ courses of MASCT, was better than those in the control group. No serious adverse events were reported in this study, suggesting that MASCT was well tolerated by the patients.

Many preclinical and clinical studies of the DC vaccine and adoptive $\mathrm{T}$ cell immunotherapy have been conducted over the last decade. The MASCT methodology combines these two therapies to improve the prognosis of HCC patients after curative treatment. In MASCT, tumor cells are destroyed through synergistic mechanisms; the DC vaccine triggers the active immune response, and the infused $\mathrm{T}$ cells evoke the passive immune response. The injection of DCs pulsed with tumor antigens followed by transferring with activated $\mathrm{T}$ cells stimulated with these DCs, may not only help to generate tumor-specific $\mathrm{T}$ cells in vivo ${ }^{13}$, but also to transplant ex vivo-prepared tumor-specific $\mathrm{T}$ cells to cancer patients ${ }^{14}$. In patients who received MASCT, T cells can respond specifically to different antigens, and efficiently activate an available $\mathrm{T}$ cell repertoire against tumor. Patients who respond better to MASCT experience a longer duration before tumor recurrence and death ${ }^{20}$. In this study, the patients who received MASCT tended to experience longer DFS and OS. This was possibly caused by the presence of DCs that were activated with multiple tumor antigens, as well as restoration of the function of exhausted T cells, and the prevention of immune escape in vivo ${ }^{25}, 26$. Furthermore, the patients who received multiple courses of MASCT showed longer DFS and OS than the other patients in this study, indicating that the chance of a cumulative immune response is enhanced by performing multiple courses of $\mathrm{MASCT}^{27}$. In addition, the effects of immunotherapy (e.g. DC 
vaccine, ACT) on time-to-event endpoints such as survival, may take several months to manifest, and can lead to the delayed separation of Kaplan-Meier survival curves ${ }^{28-30}$. This delay may explain the statistically significant difference in OS, but not in DFS, between the multiple-course MASCT subgroup and the control group in the present study.

The baseline characteristics were imbalanced. This imbalance may have favored the MASCT group, as the ages of patients in the MASCT group and in the multiple-course MASCT subgroup were significantly lower than that of the control group. In addition, it should be noticed that in the clinic, young patients tend to choose more positive treatment protocols, which perhaps favored the MASCT group in this retrospective study. Typically, older age is associated with a higher prevalence of comorbidities, liver dysfunction and serious immunological impairment. However, the role of age on treatments and outcome of HCC patients is still controversial. A demographic survey of HCC incidence and liver cancer mortality rates in the U.S. showed that the mortality rates were lower in younger men (35-49 year old) than elder men $(50+\text { years })^{31}$. Whereas, the results of propensity score analysis indicated that survival was unaffected by age after HCC treatment in some other retrospective cohort study ${ }^{32}, 33$. Thus, whether the efficacy of MASCT treatment can be affected by age still need further study. Other key baseline characteristics, including Child-Pugh score and BCLC stage, were all balanced. For curative treatment, clinical prognosis seems to be different in different modalities, especially between liver resection and $\mathrm{RF}^{34}$. Following the National Comprehensive Cancer Network (NCCN) guidelines ${ }^{23}$, the percutaneous ablation including RF, cryoablation and PEI were combined in this study because of similar clinic prognosis ${ }^{35}$. The baseline characteristics of liver resection and percutaneous ablation were also balanced. Even for age, in preplanned multivariate analysis and subgroup analysis, this imbalanced baseline characteristic was proven to have no significant impact on the effectiveness of MASCT.

Limitations of this study remain, since it is a retrospective study with a small sample size. The efficacy of MASCT in HCC patients must be further investigated. A prospective large-scale, randomized, controlled clinical trial would provide more definitive evidence on the efficacy and safety of MASCT.

\section{Conclusions}

This study is the first comprehensive retrospective review of MASCT for HCC that has been used in a clinical setting. It was demonstrated that multiple-course $(\geq 5)$ MASCT treatment can significantly prolong OS in patients who have undergone curative treatment for HCC, and that it is well-tolerated by patients. This finding also proves that the cumulative effect of MASCT immunotherapy is necessary to prolong patient survival. Thus, MASCT may be a valuable and safe therapeutic strategy for HCC patients to prolong survival time and improve prognosis. Further clinical studies on the benefits of MASCT should be performed in the near future.

\section{Abbreviations}

MASCT: multiple antigen stimulating cellular therapy; HBV: hepatitis B virus; OS: overall survival; DFS: disease-free survival; HR: hazard ratio; CI: confidence interval; KPS: Karnofsky Performance Status; BCLC: Barcelona Clinic Liver Cancer; ETV: entecavir; RF: radiofrequency; PEI: percutaneous ethanol injection; TACE: Transcatheter arterial chemoembolization; DC: dendritic cell; ACT: adoptive cell therapy; APC: antigen presenting cell; CAR: chimeric antigen receptor; TAA: tumor associated antigen; PBMC: peripheral blood mononuclear cell; CT: computed tomography; MRI: magnetic resonance imaging; AFP: alpha-fetoprotein; WBC: white blood cell; LYM: lymphocyte; PLT: platelet; ALP: alkaline phosphatase; GGT: gamma-glutamyl transpeptidase; TBIL: total bilirubin; ALT: alanine aminotransferase; AST: aspartate transaminase; ALB: albumin; PT: prothrombin time; AE: adverse event; SAE: serious adverse event.

\section{Supplementary Material}

Supplementary tables.

http://www.jcancer.org/v09p1385s1.pdf

\section{Acknowledgements}

We thank Feng Xiaorong, Chen Huaiyu, Liu Dingli and everyone else who took part in this study.

\section{Authors' contributions}

JH: conceived and supervised the project. LL: general guider of the study. YH: collected patient information, performed statistical analysis and drafted the manuscript. YG, JC, XH, XL, YK, XZ, XZ designed and performed the study and analyzed data. All authors have read and approved the final manuscript.

\section{Funding}

This research was supported by: The Key Research Fund from the National Key Basic Research Program of China (973 Program, No.2015CB554000), and Guangzhou Science and Technology Plan Project (201607020019 and 201604020002). 


\section{Availability of data and materials}

The datasets supporting the conclusions of this article are available on request.

\section{Consent for publication}

All patients signed forms to provide informed consent before MASCT was administered.

\section{Ethics approval and consent to participate}

This study protocol conformed to the ethical guidelines of the 1975 Declaration of Helsinki. The collection of clinical data was approved by the Ethics Committee of Nanfang hospital, Southern Medical University, Guangzhou, China.

\section{Competing Interests}

The authors have declared that no competing interest exists.

\section{References}

1. Lai CL, Ratziu V, Yuen MF, et al. Viral hepatitis B. Lancet. 2003; 362(9401): 2089-94.

2. Fan JH, Wang JB, Jiang $Y$, et al. Attributable causes of liver cancer mortality and incidence in china. Asian Pac. J. Cancer Prev. 2013; 14(12): 7251-6.

3. Livraghi T, Meloni F, Di Stasi M, et al. Sustained complete response and complications rates after radiofrequency ablation of very early hepatocellular carcinoma in cirrhosis: Is resection still the treatment of choice? Hepatology. 2008; 47(1): 82-9.

4. Nathan H, Schulick RD, Choti MA, et al. Predictors of survival after resection of early hepatocellular carcinoma. Ann. Surg. 2009; 249(5): 799-805.

5. Roayaie S, Obeidat K, Sposito C, et al. Resection of hepatocellular cancer $\leq 2$ $\mathrm{cm}$ : results from two Western centers. Hepatology. 2013; 57(4): 1426-35.

6. Kluger MD, Salceda JA, Laurent A, et al. Liver resection for hepatocellular carcinoma in 313 Western patients: tumor biology and underlying liver rather than tumor size drive prognosis. J. Hepatol. 2015; 62(5): 1131-40.

7. Forner A, Llovet JM, Bruix J. Hepatocellular carcinoma. Lancet. 2012; 379: 1245-55.

8. Peng BG, He Q, Li JP, et al. Adjuvant transcatheter arterial chemoembolization improves efficacy of hepatectomy for patients with hepatocellular carcinoma and portal vein tumor thrombus. Am. J. Surg. 2009; 198(3): 313-8.

9. Zhong C, Guo RP, Li JQ, et al. A randomized controlled trial of hepatectomy with adjuvant transcatheter arterial chemoembolization versus hepatectomy alone for Stage III A hepatocellular carcinoma. J. Cancer Res. Clin. Oncol. 2009; 135(10): 1437-45.

10. Li F, Guo Z, Zhang Y, Wang $\mathrm{H}$, et al. Postoperative adjuvant arterial chemoembolization improves the survival of hepatitis $B$ virus-related hepatocellular carcinoma: a retrospective control study. Ir. J. Med. Sci. 2015; 184(4): 753-9.

11. Liao $\mathrm{Y}, \mathrm{Zheng} \mathrm{Y}, \mathrm{He} \mathrm{W}$, et al. Sorafenib therapy following resection prolongs disease-free survival in patients with advanced hepatocellular carcinoma at a high risk of recurrence. Oncol. Lett. 2017; 13(2): 984-992.

12. De Simone P, Crocetti L, Pezzati D, et al. Efficacy and safety of combination therapy with everolimus and sorafenib for recurrence of hepatocellular carcinoma after liver transplantation. Transplant Proc. 2014; 46(1): 241-4.

13. Palucka K, Banchereau J. Dendritic-cell-based therapeutic cancer vaccines. Immunity. 2013; 39(1): 38-48.

14. Restifo NP, Dudley ME, Rosenberg SA. Adoptive immunotherapy for cancer: harnessing the T cell response. Nat. Rev. Immunol. 2012; 12(4): 269-81.

15. Gross S, Erdmann M, Haendle I, et al. Twelve-year survival and immune correlates in dendritic cell-vaccinated melanoma patients. JCI Insight. 2017; 2(8).

16. Wong JL, Berk E, Edwards RP, et al. IL-18-primed helper NK cells collaborate with dendritic cells to promote recruitment of effector CD8+ T cells to the tumor microenvironment. Cancer Res. 2013; 73(15): 4653-62.

17. Boudewijns $\mathrm{S}$, Bol KF, Schreibelt G, et al. Adjuvant dendritic cell vaccination induces tumor-specific immune responses in the majority of stage III melanoma patients. Oncoimmunology. 2016; 5(7): e1191732.

18. Kingwell K. CAR T therapies drive into new terrain. Nat. Rev. Drug. Discov. 2017; 16(5): 301-4.

19. Feng K, Liu Y, Guo Y, et al. Phase I study of chimeric antigen receptor modified $\mathrm{T}$ cells in treating HER2-positive advanced biliary tract cancers and pancreatic cancers. Protein Cell. 2017; [Epub ahead of print].
20. Han $Y, W u ~ Y, Y a n g ~ C$, et al. Dynamic and specific immune responses against multiple tumor antigens were elicited in patients with hepatocellular carcinoma after cell-based immunotherapy. J. Transl. Med. 2017; 15(1): 64.

21. European Association for Study of Liver; European Organisation for Research and Treatment of Cancer. EASL-EORTC clinical practice guidelines: management of hepatocellular carcinoma. Eur. J. Cancer. 2012; 48: 599-641.

22. Thomas MB, Jaffe D, Choti MM, et al. Hepatocellular carcinoma: consensus recommendations of the National Cancer Institute Clinical Trials Planning Meeting. J. Clin. Oncol. 2010; 28(25): 3994-4005.

23. [Internet] National Comprehensive Cancer Network. NCCN clinical practice guidelines in oncology, hepatobiliary cancers 2015. https://www.nccn.org/professionals/physician_gls/pdf/hepatobiliary.pdf

24. Trotti A, Colevas AD, Setser A, et al. CTCAE v3.0: development of a comprehensive grading system for the adverse effects of cancer treatment. Semin. Radiat. Oncol. 2003; 13: 176-81.

25. Tan AT, Koh S, Goh W, et al. A longitudinal analysis of innate and adaptive immune profile during hepatic flares in chronic hepatitis B. J. Hepatol. 2010; 52(3): 330-9.

26. Bertoletti A, Brunetto $M$, Maini $M K$, et al. $T$ cell receptor-therapy in HBV-related hepatocellularcarcinoma. Oncoimmunology. 2015; 4(6): e1008354.

27. Boudewijns S, Bol KF, Schreibelt $G$, et al. Adjuvant dendritic cell vaccination induces tumor-specific immune responses in the majority of stage III melanoma patients. Oncoimmunology. 2016; 5(7): e1191732.

28. Finke, L. H, Wentworth K, Blumenstein B. et al. Lessons from randomized phase III studies with active cancer immunotherapies--outcomes from the 2006 meeting of the Cancer Vaccine Consortium (CVC). Vaccine. 2007; 25 Suppl 2: B97-B109.

29. Hoos A, Eggermont AM, Janetzki S, et al. Improved endpoints for cancer immunotherapy trials. J. Natl. Cancer Inst. 2010; 102(18): 1388-97.

30. Hodi FS, O'Day SJ, McDermott DF, et al. Improved survival with ipilimumab in patients with metastatic melanoma. N. Engl. J. Med. 2010; 363(8): 711-23.

31. Altekruse SF, Henley S, Cucinelli JE3, et al. Changing hepatocellular carcinoma incidence and liver cancer mortality rates in the United States. Am J Gastroenterol. 2014; 109(4):542-53.

32. Mirici-Cappa F, Gramenzi A, Santi V, et al. Treatments for hepatocellular carcinoma in elderly patients are as effective as in younger patients: a 20-year multicentre experience. Gut. 2010; 59(3): 387-96.

33. Guo H, Wu T, Lu Q, et al. Hepatocellular carcinoma in elderly: Clinical characteristics, treatments and outcomes compared with younger adults. PLoS One. 2017;12(9):e0184160.

34. Wang C, Wang $\mathrm{H}$, Yang $\mathrm{W}$, et al. Multicenter randomized controlled trial of percutaneous cryoablation versus radiofrequency ablation in hepatocellular carcinoma. Hepatology. 2015; 61(5): 1579-90.

35. Feng K, Yan J, Li X, et al. A randomized controlled trial of radiofrequency ablation and surgical resection in the treatment of small hepatocellular carcinoma. J. Hepatol. 2012; 57(4): 794-802. 